\title{
PAPER
}

\section{Complications after gamma knife radiosurgery for benign meningiomas}

\author{
J H Chang, J W Chang, J Y Choi, Y G Park, S S Chung
}

J Neurol Neurosurg Psychiatry 2003;74:226-230

See end of article for authors' affiliations

.....................

Correspondence to: Professor Y G Park Department of

Neurosurgery, Yonse University College of

Medicine, CPO Box 8044

Seoul, Korea:

ygpark@umc.yonsei.ac.kr

Received 15 April 2002

In final revised form 27

September 2002

Accepted

23 October 2002

\begin{abstract}
Objectives: To analyse the results of gamma knife radiosurgery (GKS) for the trreatment of intracranial meningiomas and to assess possible factors related to the outcome and complications of such treatment.

Methods: The authors retrospectively reviewed the clinical and radiological data of 179 patients (194 lesions) treated with GKS for meningiomas between May 1992 and October 2000. The mean follow up duration was 37.3 months (range 6.4 to 86.3 months). The study determined the correlation between radiosurgical outcome including imaging changes after GKS and multiple factors such as tumour location and size, patient characteristics, venous sinus status, pre-GKS degree of oedema, other treatment modalities, and radiosurgical parameters.

Results: The radiological control rate was $97.1 \%$. Magnetic resonance imaging (MRI) showed complications after GKS in 35 lesions (25.0\%) among the 140 lesions followed up with MRI. Complications were divided into peritumorous imaging changes (33 lesions; $23.6 \%$ ) and transient cranial nerve dysfunction (two lesions; $1.4 \%$ ). Radiation induced imaging changes were seen mostly in convexity, parasagittal, and falx meningiomas that were deeply embedded in the cortex. About $60 \%$ of these were asymptomatic and the overall rate of symptomatic imaging changes was $9.3 \%$. Neurological deficit related to imaging changes developed in only three patients, and all the symptoms were transient.

Conclusion: GKS for intracranial meningiomas seems to be a safe and effective treatment. However, meningiomas of the convexity, parasagittal region, or falx cerebri have a higher incidence of peritumorous imaging changes after GKS than those of the skull base. Therefore, the use of GKS needs to be considered very cautiously in cerebral hemispheric meningiomas, taking into consideration patient age and general condition, tumour size and location, pattern of cortical embedding, relation between the tumour and venous sinuses, presenting symptoms, and patient preference.
\end{abstract}

A though more than $90 \%$ of intracranial meningiomas are histopathologically benign, they frequently recur if removal is incomplete. ${ }^{1-9}$ While surgical approaches and techniques for these tumours have undergone continual development, their complete removal remains difficult, especially for parasellar, cavernous, orbital, and petroclival meningiomas and meningiomas adjacent to the major venous sinuses. As a result, the role of radiosurgery has been increasing in importance as a primary or adjuvant treatment modality. ${ }^{1-6-16}$ Meningiomas are suitable for radiosurgery, because they are encapsulated and often small at time of diagnosis, usually do not invade the brain, and are well defined by computed tomography or magnetic resonance imaging (MRI). These features allow steep radiation fall off and precise definition of target volume. Because their blood supply usually arises from the dura, it can be included in the treatment volume, and this can cause tumour infarction and necrosis.

Recent advances in high resolution imaging technique and sophisticated three dimensional workstations have improved the accuracy of dose planning during gamma knife radiosurgery (GKS). ${ }^{6}$ Nevertheless, there have been major and minor complications after GKS, so proper selection of meningiomas for GKS is still very important. We, therefore, analysed the radiosurgical outcomes and complications resulting from treatment of benign meningiomas with GKS and further evaluated potential aetiologies of treatment complications.

\section{METHODS}

Between May 1992 and October 2000, 187 patients with meningiomas were treated with GKS at our institution. This group

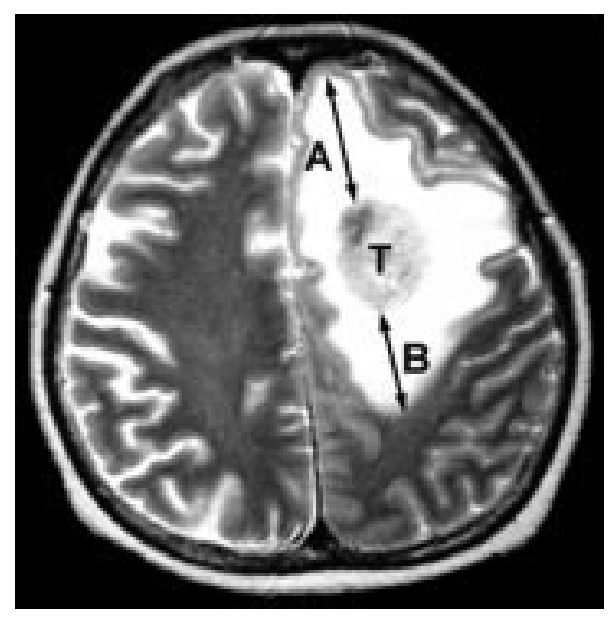

Figure 1 The degree of peritumorous oedema: the diameter of oedema $(A+B$ in $\mathrm{cm})$ from the tumour margin (T) on T2WI MRI.

represented $28.5 \%$ of all brain tumours treated with GKS. After we excluded one atypical meningioma and seven malignant meningiomas from this study, we retrospectively reviewed the clinical and radiological data in 194 lesions of 179 patients treated with GKS for intracranial meningiomas.

Abbreviations: GKS, gamma knife radiosurgery; MRI, magnetic resonance imaging 
Table 1 Summary of failed cases after GKS $(n=4)$

\begin{tabular}{lllllll}
\hline No & Sex/age $(y)$ & Location & Max/margin (Gy) & Volume (cc) & Recur time (m) & Result \\
\hline 1 & M/37 & Convexity & $31 / 15.5$ & 21 & 81 & Remarks \\
2 & M/39 & Petroclival & $25 / 12.5$ & 5 & 63 & Re-GKS \\
3 & M/65 & Convexity & $32 / 16$ & 5.5 & 28 & Observation \\
4 & F/63 & Parasagittal & $30 / 13.5$ & 26 & 11 & Multiple \\
\hline
\end{tabular}

GKS, gamma knife radiosurgery; $C / R$, craniotomy and removal of tumour.

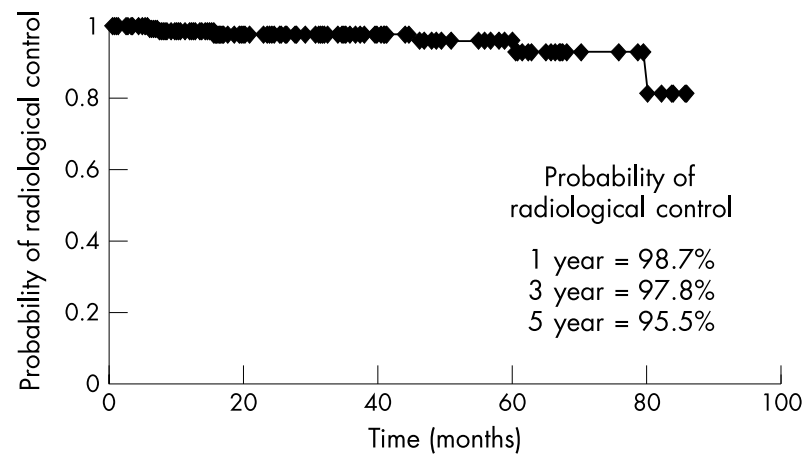

Figure 2 Cumulative events curve by Kaplan-Meier analysis for radiological control of meningiomas after GKS.

There were nine patients with multiple meningiomas. We used the KULA dose planning system (version 5.4, Elekta, Sweden; 164 lesions) until September 1999, when we began using the GammaPlan (version 5.30, Elekta, Sweden; 30 lesions).

Among the 194 lesions, 140 lesions (72.2\%) were radiologically followed up for more than six months with MRI and the mean follow up duration was 37.3 months (range 6.4 to 86.3 months). When the volume of a tumour decreased by more than $20 \%$ of pre-GKS volume on follow up MRI, it was defined as shrinkage and represented as the volume response of a tumour to GKS. When it changed by less than $20 \%$, or increased by more than $20 \%$, it was defined as stasis or enlargement, respectively. Shrinkage and stasis were defined as radiological controls. The degree of peritumorous oedema was graded by first subtracting the maximal diameter $(\mathrm{cm})$ of the tumour from that of the peritumorous oedema on the T2 weighted MR image and then rounding off the result to the nearest whole number (fig 1 ). The grade 0 indicates that there was no peritumorous oedema and increasing grade indicates increasing severity of the peritumorous oedema.

A Cox proportional hazards model was used to evaluate the correlation between radiosurgical outcomes and imaging changes after GKS and various factors such as tumour location, lobar location and size, patient age, sex, and presenting symptom, relation to major venous sinus, pre-GKS degree of oedema, treatment modality, and various radiosurgical parameters (maximum tumour dose, tumour margin dose, and type of software used for dose planning). Significance was assigned as p less than 0.05 .

\section{RESULTS}

\section{Patient characteristics}

The mean age of the patients at the time of radiosurgery was 50.4 years (range 7.4 to 82.2 years). The ratio of male to female patients was 1:3.5 (40 male and 139 female patients). The most common presenting symptoms were headache in 55 patients $(30.7 \%)$, trigeminal neuralgia in $18(10.1 \%)$, and visual disturbance in $13(7.3 \%)$. In 49 patients $(27.4 \%)$, the meningiomas were incidentally detected.

\section{Radiological findings}

Most of the tumours were located in the skull base $(n=112$; $57.7 \%)$, and cerebral hemispheres $(n=72 ; 37.1 \%)$. The rest
Table 2 Postradiosurgical imaging changes

\begin{tabular}{lll}
\hline Location & $\begin{array}{l}\text { GKS induced IC } \\
(\%)\end{array}$ & $\begin{array}{l}\text { Symptomatic IC } \\
(\%)\end{array}$ \\
\hline Cerebral hemispheric & $26 / 52(50.0)$ & $11 / 52(21.2)$ \\
$\quad$ Convexity & $12 / 22(54.5)$ & $4 / 22(18.2)$ \\
$\quad$ Parasagittal & $11 / 15(73.3)$ & $6 / 15(40)$ \\
$\quad$ Falx & $3 / 15(20.0)$ & $1 / 15(6.7)$ \\
Skull base & $4 / 79(5.1)$ & $1 / 79(1.3)$ \\
Other & $3 / 9(33.3)$ & $1 / 9(11.1)$ \\
Total & $33 / 140(23.6)^{*}$ & $13 / 140(9.3)^{*}$ \\
\hline
\end{tabular}

*MRI follow up (>6 months) in 140/194 lesions (72.2\%). GKS, gamma knife radiosurgery; IC, imaging changes.

were in the ventricles, pineal region, or sylvian fissure $(n=10$; $5.2 \%$ ). Cerebral hemispheric meningiomas included convexity, parasagittal, and falx meningiomas. Among the 72 hemispheric meningiomas, $42(58.3 \%)$ were located in the frontal region, $18(25.0 \%)$ in the parietal region, four $(5.6 \%)$ in the occipital region, three $(4.2 \%)$ in the temporal region, and five $(6.9 \%)$ in the cerebellar convexity.

The relation between tumour and major venous sinus was classified into three groups: complete occlusion of venous sinus due to tumour invasion $(\mathrm{n}=2,1.0 \%)$; partial occlusion of venous sinus $(n=21,10.8 \%)$; and no venous sinus invasion $(\mathrm{n}=171,88.1 \%)$.

Pre-GKS peritumorous oedema, grade 1 to 6 , was found in 26 lesions (13.4\%).

\section{Treatment modalities}

Of the 179 patients, GKS was performed as a primary treatment in $109(60.9 \%)$ and as an adjuvant treatment for residual or recurrent lesions after surgical resection in the other $70(39.1 \%)$. No patient received conventional radiation therapy.

\section{Radiosurgical parameters}

The volume of tumour was estimated by the area within the isodose curve during dose planning. The mean volume of tumours was $10.1 \mathrm{cc}$ (range 0.6 to $45 \mathrm{cc}$ ). The tumour was covered within $40 \%$ to $90 \%$ (mean $50.5 \%$ ) of the isodose curve; the mean tumour margin dose was 15.1 Gy (range 9.5 to 24.5 Gy); the mean maximum tumour dose was 30.0 Gy (range 19 to $45 \mathrm{~Gy}$ ); and the mean number of isocentres was 6.3 (range 1 to 15$)$.

\section{Radiological response}

Of the 140 lesions (72.2\%) followed up for more than six months with MRI, shrinkage was found in 77 (55.0\%), stasis in $59(42.1 \%)$, and enlargement in four $(2.9 \%)$. Radiological control, either shrinkage or stasis was achieved in 136 of 140 lesions, giving a radiological control rate of $97.1 \%$. The probability of radiological control after GKS was analysed with Kaplan-Meier analysis (fig 2). We have provided additional data on the four intracranial meningiomas that failed GKS (table 1). We were unable to identify statistically significant factors related to radiological control of meningiomas or failure of treatment after GKS. 

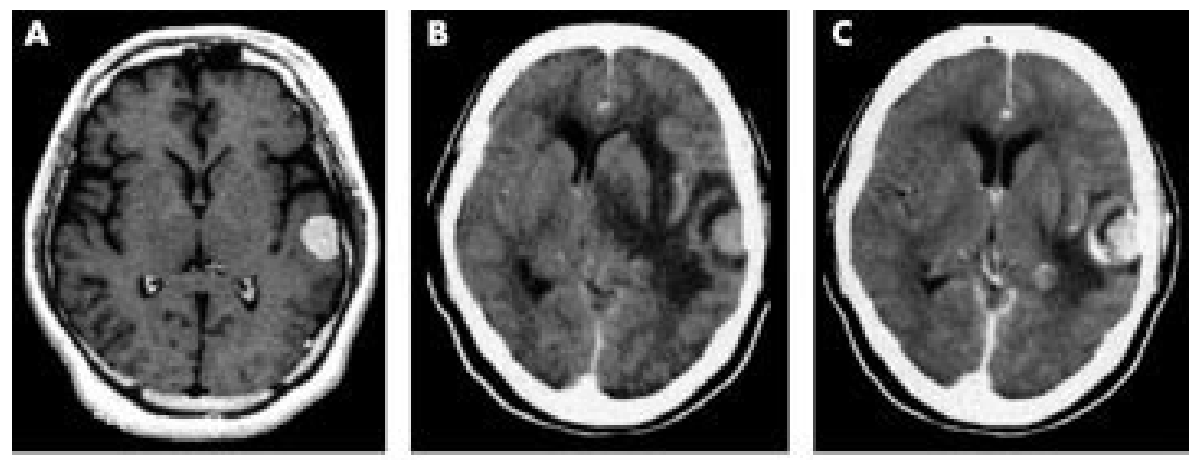

Figure 3 (A) Pre-radiosurgical MRI showing an incidental convexity meningioma. (B-F) Follow up computed tomography $(4,5,8,12$, and 16 months after GKS, respectively) showing recurrent peritumorous imaging changes. The imaging changes responded well to corticosteroid treatment. Sixteen months after GKS the imaging changes were completely improved and the tumour was slightly shrunken.
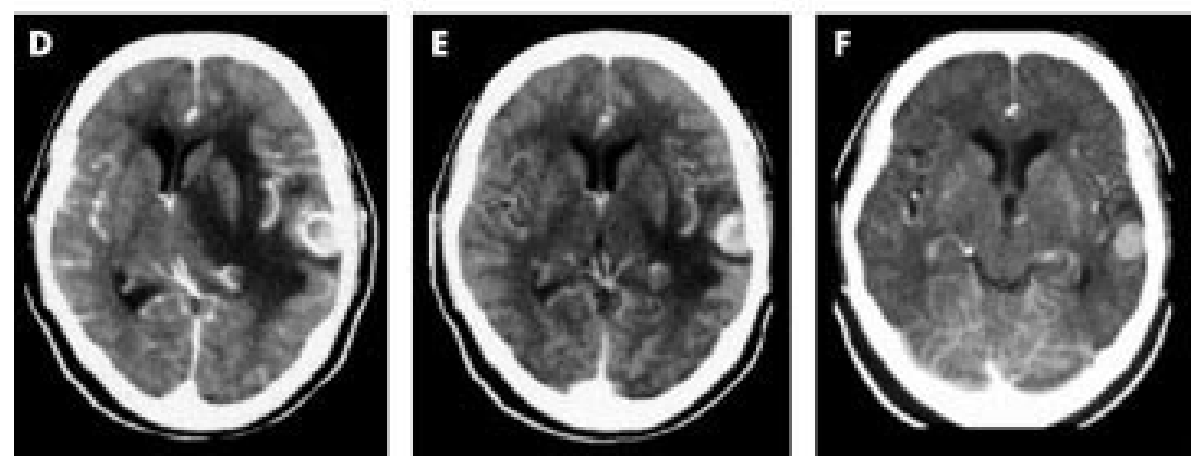

Cerebral hemisphere

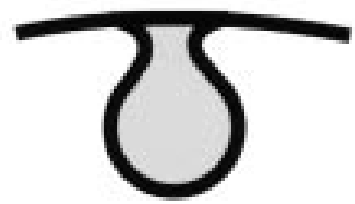

Skull base

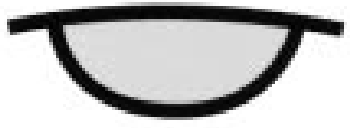

Figure 4 Difference of growth pattern between skull base meningiomas and cerebral hemispheric meningiomas. While the first usually expand laterally along cisterns, the second such as convexity, parasagittal, and falx meningiomas grow deeply embedding into the cortex.
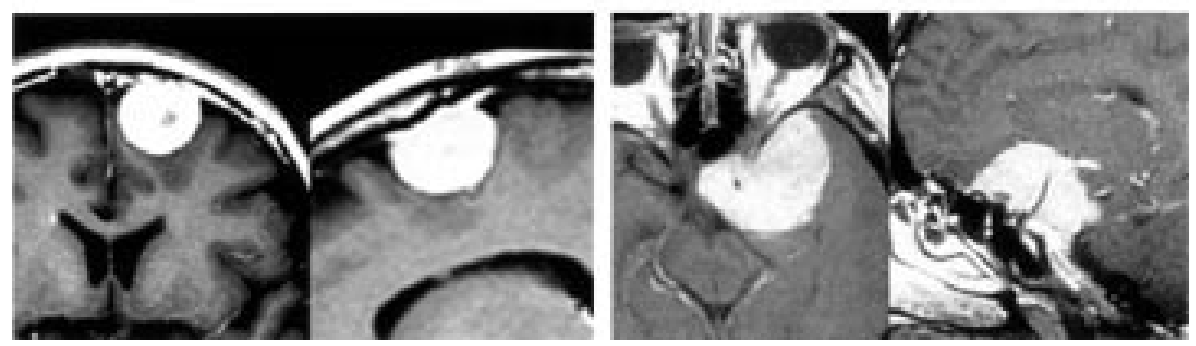

\section{Complications}

Complications after GKS for meningiomas were found in 35 lesions $(25.0 \%)$ among the 140 lesions followed up with MRI. Complications were divided into peritumorous imaging changes on follow up MRI in 33 lesions (23.6\%) and transient cranial nerve dysfunction in the other two (1.4\%). Peritumourous imaging changes included newly developed imaging changes and aggravation of pre-existing imaging changes on follow up MRI after GKS (fig 3). Among the 33 peritumorous lesions, $13(39.4 \%)$ produced either headaches, caused by increased intracranial pressure (six lesions); seizures (four lesions); or other neurological deficits (three lesions). The symptoms were all transient. The overall rate of symptomatic oedema was $9.3 \%$. Imaging changes developed at a mean of 7.8 months (range 2.8 to 48.9 months) after GKS and were sustained for 13.5 months (range 3.0 to 28.0 months). Imaging changes were evident in four $(5.1 \%)$ of the 79 skull base meningiomas and $26(50.0 \%)$ of the 52 hemispheric meningiomas. The symptomatic imaging changes occurred more frequently in hemispheric meningiomas (table 2).
The cranial nerve dysfunction seen in two patients was also transient. The first patient had undergone GKS for a cavernous meningioma extending to the orbital fossa. The tumour margin dose was $16.5 \mathrm{~Gy}$ and the estimated dose to the lateral wall of the cavernous sinus was 16.5 Gy. Decreased hemifacial sensation developed six months after GKS and was present for one month. The second patient with a cavernous meningioma had undergone GKS with 13.3 Gy of tumour margin dose. The estimated dose to the cavernous sinus was 18.6 Gy. The patient developed an abducens nerve palsy at 18 months after GKS but improved two months later.

We analysed the factors related to peritumorous imaging changes on MRI after GKS. In univariate analysis, tumour location $(p<0.001)$, maximum tumour dose $(p=0.0002)$, and tumour margin dose $(\mathrm{p}=0.037)$ were significantly related to imaging changes after GKS. However, in multivariate analysis, only tumour location was significant. Cerebral hemispheric meningiomas had a higher rate of peritumorous imaging changes after GKS than tumours at other locations such as the skull base, ventricles, pineal region, or sylvian fissure. 


\section{DISCUSSION}

The role of radiosurgery has been gaining importance as a primary or adjuvant treatment modality for meningiomas and many authors have reported excellent outcome for meningiomas treated with GKS. ${ }^{13-6} 810-19$ We identified peritumorous imaging changes on MRI after GKS in $23.6 \%$ of lesions. Most of these did not exhibit any symptoms, and all of the symptomatic patients $(9.3 \%)$ completely recovered. The detailed mechanism and pathophysiology of perilesional imaging changes after radiosurgery are unclear. Most peritumorous imaging changes are believed to be attributable to vasogenic oedema. Whether this is attributable to vascular endothelial growth factor (VEGF)/vascular permeability factor (VPF), an angiogenic growth factor present in most meningiomas, ${ }^{20-24}$ or radiation injury to the vasculature is unknown.

There have been many reports about the risk factors for oedema development after radiosurgery. Kalapurakal et al reported that parasagittal location, presence of pretreatment oedema, sagittal sinus occlusion, and radiation doses above 6 Gy per fraction, were significant risk factors for oedema development after radiation therapy. ${ }^{25}$ In a multicentre review, Kondziolka et al identified 18 of 185 patients with symptomatic oedema that required treatment after GKS. ${ }^{3}$ They suggested that the presence of a pre-GKS neurological deficit was the most important factor for subsequent symptomatic oedema and that the tumour margin dose was not related to onset of oedema after GKS. Several groups have also reported that non-skull base meningiomas have a higher risk of peritumorous oedema after GKS. ${ }^{8}{ }^{17}{ }^{26}$ Ganz et al suggested that oedema developed preferentially in non-basal tumours, especially those around the midline and sagittal sinus and in cases with a margin tumour dose more than $18 \mathrm{~Gy} .{ }^{17}$ Besides the factors described above, patient age, histological finding, tumour growth, oestrogen and progesterone receptor positivity, and presence of secretory and excretory granules by electron microscopy were suggested as possible risk factors for peritumorous oedema. ${ }^{182526}$

In our study, post-GKS peritumorous imaging changes developed mostly in cerebral hemispheric meningiomas of the convexity, parasagittal region, or falx cerebri. In univariate analysis, tumour location (skull base or cerebral hemisphere), maximum tumour dose, and tumour margin dose were correlated with the occurrence of imaging changes after GKS. However, in multivariate analysis tumour location was the only significant factor. Other factors reported as significant by other investigators, such as pre-existing oedema, occlusion of venous sinus, or patient age, did not influence the occurrence of imaging changes after GKS.

The influence of tumour location on the rate of occurrence of imaging changes-that is, peritumorous oedema, seems to be related to the different growth patterns of skull base and hemispheric meningiomas (fig 4). While skull base meningiomas spread laterally along the cistern, maintaining a wide base on the dura at the base of the skull, cerebral hemispheric meningiomas are embedded in the brain, and, therefore, have a wider contact area with the brain parenchyma than skull base meningiomas. Unlike skull base meningiomas, which are surrounded by cisterns, hemispheric meningiomas lack any intervening arachnoid or cerbral spinal fluid barrier between the tumour and cortical surface. Therefore, the brain parenchyma surrounding hemispheric meningiomas seems to be more easily affected by the oedema fluid.

GKS has played an important part in the treatment of skull base meningiomas, because the surgical approach carries a high risk of complications and recurrence. ${ }^{14-69-1526}$ Skull base meningiomas were over-represented in our study of GKS treated meningiomas $(57.7 \%)$, compared with their prevalence in the whole meningioma population. There are many cranial nerves and critical structures around the skull base, especially the cavernous sinus, and careful dose planning is required. ${ }^{6} 12141527$
The most critical structure is the optic pathway, which many authors believe tolerates a radiation dose of less than 8 to 10 Gy. ${ }^{4142728}$ Girkin et al reported that optic neuropathy developed 7 to 30 months after GKS for perichiasmal tumours as an abrupt change in the visual field to a homonymous hemianopic defect. ${ }^{29}$ MRI showed swelling and contrast enhancement of the affected portion of the visual apparatus. They suggested that risk factors for the development of radiation optic neuropathy after GKS included dose to the optic apparatus of greater than $8 \mathrm{~Gy}$, previous conventional radiation therapy, pre-existing anterior visual pathway dysfunction secondary to previous surgery or tumour compression, treatment plans based on computed tomography rather than MRI, large tumour volume, and treatment isocentres within $5 \mathrm{~mm}$ of the anterior visual pathways. Kondziolka et al insisted that only a $1 \mathrm{~mm}$ gap between tumour margin and the chiasm might be necessary, if this proximity occurred only at one point, as verified with current high resolution imaging techniques. ${ }^{4}$ Another report suggested that even more than 10 Gy was safe for the optic apparatus. ${ }^{12}$ However, we believe that the radiation dose to the optic pathway should not exceed 10 Gy. In our studies, the dose to the optic pathway was less than 9.6 Gy and there were no visual complications. Recent advances in neuroradiological imaging and radiosurgical technique have permitted effective and safe control for tumours with a closer contact to the optic pathway. ${ }^{6}$

Beyond the optic pathway, attention also should be paid to the radiation dose to the cranial nerves running along the wall of the cavernous sinus, Meckel's cave, hypothalamus, pituitary gland and stalk, and brain stem. ${ }^{612142728}$ We previously suggested the optimal radiation dose for critical juxtasellar structures. ${ }^{27}$ While 48 patients with radiosurgically treated juxtasellar tumours with a dose of less than 16 Gy to the cavernous sinus had no cranial nerve deficits, two other patients with a dose of more than 16 Gy experienced cranial nerve dysfunction. In this study, both of the patients with transient cranial nerve dysfunctions, received more than 16.0 Gy to the cranial nerves.

While 12 Gy to 15 Gy has been recommended as the minimal dose for the meningioma margin, ${ }^{68121517}$ low dose radiosurgery has been tried by several groups to avoid complications associated with radiation therapy. ${ }^{810} 19$ While a lower dose may be sufficient for effective tumour control with decreased complication rate, the follow up durations of these low dose radiosurgery studies are comparatively short. The minimal dose for tumour margin remains controversial and longer follow up studies are needed.

In our study, we identified four failed cases of meningioma after GKS. One patient had undergone GKS for a residual convexity meningioma after surgical resection. The tumour had nearly disappeared three years after GKS, but recurred on follow up MRI taken at seven years after GKS. The recurrence was thought to arise from the peritumorous dural extension not included within a proper isodose curve. We believe it is essential to irradiate the peritumorous dural extension optimally to achieve long term tumour control.

In conclusion, GKS for intracranial meningiomas is a safe and effective form of primary or adjuvant treatment after incomplete surgical resection. However, patients with cerebral hemispheric meningiomas of convexity, parasagittal region, or falx cerebri have a higher incidence of peritumorous imaging changes after GKS than those with skull base meningiomas. These imaging changes are probably explained by postradiosurgical peritumorous oedema. Therefore, patient age and general condition, tumour size and location, pattern of cortical embedding, relation between the tumour and venous sinuses, presenting symptoms and patient preference need to be considered when treating hemispheric meningiomas with GKS.

\section{ACKNOWLEDGEMENT}

We thank Drs Steven M Rothman and Michael Wong (Department of Neurology, Washington University School of Medicine, St Louis, MO) for helpful discussions. 


\section{Authors' affiliations}

J H Chang, J W Chang, J Y Choi, Y G Park, S S Chung, Department of Neurosurgery, Brain Research Institute, and BK 21 Project for Medical Science, Yonsei University College of Medicine, Seoul, Korea

Competing interests: none declared.

\section{REFERENCES}

1 Aichholzer M, Bertalanffy A, Dietrich W, et al. Gamma knife radiosurgery of skull base meningiomas. Acta Neurochir (Wien) 2000;142:647-53.

2 Condra KS, Buatti JM, Mendenhall WM, et al. Benign meningiomas: primary treatment selection affects survival. Int J Radiat Oncol Biol Phys 1997;39:427-36.

3 Kondziolka D, Flickinger JC, Perez B, et al. Judicious resection and/or radiosurgery for parasagittal meningiomas: outcomes from a multicenter review. Neurosurgery 1998;43:405-14.

4 Kondziolka D, Levy El, Niranjan A, et al. Long-term outcomes after meningioma radiosurgery: physician and patient perspectives. $J$ Neurosurg 1999:91:44-50.

5 Kondziolka D, Lunsford LD, Coffey RJ, et al. Gamma knife radiosurgery of meningiomas. Stereotact Funct Neurosurg 1991;57:11-21.

6 Liscak R, Simonova G, Vymazal J, et al. Gamma knife radiosurgery of meningiomas in the cavernous sinus region. Acta Neurochir (Wien) 1999;141:473-80.

7 Mirimanoff RO, Dosoretz DE, Linggood RM, et al. Meningioma: analysis of recurrence and progression following neurosurgical resection. J Neurosurg 1985;62:18-24.

8 Pan DHC, Guo WY, Chang YC, et al. The effectiveness and factors related to treatment results of Gamma Knife radiosurgery for meningiomas. Stereotact Funct Neurosurg 1998;70 (suppl 1):19-32.

9 Sekhar LN, Jannetta PJ, Burkhart LE, et al. Meningiomas involving the clivus: a six-year experience with 41 patients. Neurosurgery 1990;27:764-81

10 Iwai Y, Yamanaka K, Yasui T, et al. Gamma knife surgery for skull base meningiomas. The effectiveness of low-dose treatment. Surg Neurol 1999;52:40-5

11 Kobayashi T, Kida Y, Mori Y. Long-term results of stereotactic gamma radiosurgery of meningiomas. Surg Neurol 2001;55:325-31.

12 Morita A, Coffey RJ, Foote RL, et al. Risk of injury to cranial nerves after gamma knife radiosurgery for skull base meningiomas: experience in 88 patients. J Neurosurg 1999;90:42-9.

13 Muthukumar N, Kondziolka D, Lunsford LD, et al. Stereotactic radiosurgery for anterior foramen magnum meningiomas. Surg Neurol 1999:51:268-73.
14 Pendl G, Schrottner O, Eustacchio S, et al. Stereotactic radiosurgery of skull base meningiomas. Minim Invas Neurosurg 1997;40:87-90.

15 Shin $M$, Kurita H, Sasaki T, et al. Analysis of treatment outcome after stereotactic radiosurgery for cavernous sinus meningiomas. J Neurosurg 2001;95:435-9.

16 Stafford SL, Pollock BE, Foote RL, et al. Meningioma radiosurgery: tumor control, outcomes, and complications among 190 consecutive patients. Neurosurgery 2001:49:1029-38.

17 Ganz JC, Schrottner O, Pendl G. Radiation-induced edema after gamma knife treatment for meningiomas. Stereotact Funct Neurosurg 1996;66 (suppl 1):129-33.

18 Nakamura S, Hiyama H, Arai K, et al. Gamma knife radiosurgery for meningiomas: four cases of radiation-induced edema. Stereotact Funct Neurosurg 1996;66 (suppl 1):142-5.

19 Nakaya K, Hayashi M, Nakamura S, et al. Low-dose radiosurgery for meningiomas. Stereotact Funct Neurosurg 1999;72 (suppl 1):67-72.

20 Bitzer M, Opitz H, Popp J, et al. Angiogenesis and brain oedema in intracranial meningiomas: influence of vascular endothelial growth factor. Acta Neurochir (Wien) 1998;140:333-40.

21 Goldman CK, Bharara S, Palmer CA, et al. Brain edema in meningiomas is associated with increased vascular endothelial growth factor expression. Neurosurgery 1997;40: 1269-77.

22 Kalkanis SN, Carroll RS, Zhang J, et al. Correlation of vascular endothelial growth factor messenger RNA expression with peritumora vasogenic cerebral edema in meningiomas. J Neurosurg 1996:85:1095-101.

23 Provias J, Claffey K, delAguila L, et al. Meningiomas: role of vascular endothelial growth factor/vascular permeability factor in angiogenesis and peritumoral edema. Neurosurgery 1997;40:1016-26.

24 Yoshioka H, Hama S, Taniguchi E, et al. Peritumoral brain edema associated with meningioma. Cancer 1999;85:936-44.

25 Kalapurakal JA, Silverman CL, Akhtar N, et al. Intracranial meningiomas: factors that influence the development of cerebral edema after stereotactic radiosurgery and radiation therapy. Radiology 1997;204:461-5

26 Vermeulen S, Young R, Li F, et al. A comparison of single fraction radiosurgery tumor control and toxicity in the treatment of basal and nonbasal meningiomas. Stereotact Funct Neurosurg 1999;72 (suppl 1):60-6.

27 Chang JH, Chang JW, Park YG, et al. Gamma Knife radiosurgery for juxtasellar tumors. Journal of Korean Neurosurgical Society 2000;29:1345-51.

28 Ove R, Kelman S, Amin PP, et al. Preservation of visual fields after peri-sellar Gamma-Knife radiosurgery. Int J Cancer 2000;90:343-50.

29 Girkin CA, Comey CH, Lunsford LD, et al. Radiation optic neuropathy after stereotactic radiosurgery. Ophthalmology 1997;104:1634-43.

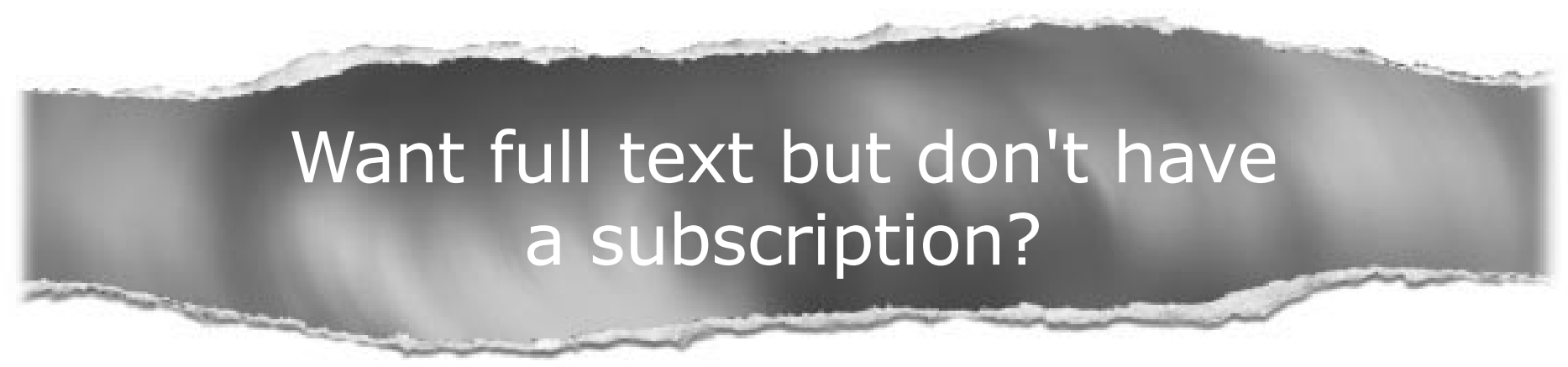

\section{Pay per view}

For just \$8 you can purchase the full text of individual articles using our secure online ordering service. You will have access to the full text of the relevant article for 48 hours during which time you may download and print the pdf file for personal use.

\section{www.jnnp.com}

